\title{
Risk Based Voltage Security Assessment
}

\author{
Hua Wan, Student Member, IEEE, James D. McCalley, Senior Member, IEEE, and Vijay Vittal, Fellow Member, IEEE
}

\begin{abstract}
The objective of this paper is to provide a risk-based approach to security assessment for a voltage stability constrained power system. It is motivated by a perception that today's deterministic reliability criteria to voltage stability assessment often results in operating restrictions that are not commensurate with the corresponding level of risk. The risk calculation provided in this paper accounts for both the future uncertainties on the system and the consequences associated with voltage collapse and violation of limits. Although the main purpose of this paper is to provide a method of evaluation, we also give an introduction to show how this reliability "leading indicator" penetrates the traditional rigid reliability boundary and how it may be used to price reliability in order to make a trade off between reliability and economics.
\end{abstract}

Index Terms-Impact, load margin sensitivity, power system security, probability, risk assessment, transmission, voltage collapse.

\section{INTRODUCTION}

$\mathbf{V}$ OLTAGE collapse typically occurs on power systems which are heavily loaded, weakened by transmission outages, or subjected to reactive power shortages. It is associated with reactive power deficiencies, and it may result in uncontrollable system-wide voltage collapse, loss of loads, and blackout. The prevailing practice in industry of avoiding voltage collapse is to maintain a deterministic reliability margin on bus voltages, reactive power requirements, transfer capabilities, or system loading levels such that the system can survive the collapse under any single component failure. The deterministic approach effectively avoids the collapse by using a conservative safety buffer against all the "dangerous" possibilities. However, it does not provide answers to the following questions:

- Risk Quantification: How safe or how risky are the current system's operating conditions?

- Trend: How does the risk change as the operating conditions are relieved or stressed?

- Security-Economy Tradeoff: How is increased risk associated with heavier use of facilities offset by the corresponding increase in benefit?

This paper attempts to answer the above questions using a cost-based risk index of voltage collapse.

\section{Problem Statement}

Fig. 1 is a typical plot of several $P-V$ curves used in analyzing a voltage constrained network. ${ }^{1}$

Manuscript received July 17, 1998; revised June 1, 1999. This work was supported by the Electrical Power Research Institute, Contract W0860401, and National Science Foundation, Grant ECS9502790.

The authors are with the Department of Electrical and Computer Engineering, Iowa State University, Ames, IA 50011 (e-mail: hwan@iastate.edu; jdm@iastate.edu; vvittal@iastate.edu).

Publisher Item Identifier S 0885-8950(00)10355-4.

${ }^{1}$ The system for which these curves are obtained is described in Section VII.

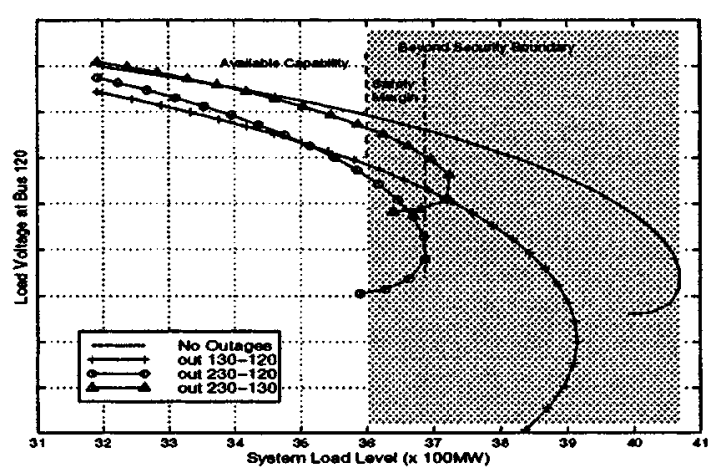

Fig. 1. $P-V$ curves at a load bus.

The solid line is a $P-V$ curve for a case without any contingencies. It shows the maximum loading capability is $4070 \mathrm{MW}$ if no contingency occurs. With the possibility of contingencies, the $P-V$ curve typically becomes more restrictive. The most constraining contingency in this case gives a maximum loadability at $3689 \mathrm{MW}$. At this operating point, the system is not safe enough because a small deviation in system conditions together with the outage of the line between buses 230 and 120 will result in a collapse of the entire system. In setting the operating guidelines, a safety margin is selected (say, $3 \%$ ), and the total capability is established. The available capability is then the distance between the current operating point and the security boundary discounted by the safety margin (Fig. 1). This procedure does not, however, provide any indication of either the degree of safety or the degree of risk associated with the operating level.

We wish to develop a risk index for voltage insecurity that provides a quantitative justification of system reliability in terms of the system economics.

\section{A. Assumptions}

- We invoke the assumption usually made for security assessment, i.e., a short-term operating condition is given. The objective of this paper is to determine the "risk" of voltage insecurity under this operating condition.

- The given operating condition has strong correlation with the condition in the near future so that we can predict the expectation $^{2}$ of the future condition very well, and that the variation of the future condition is small and some linear approximations are valid.

- The variation of the future condition away from its expectation, except for the contingencies, is due to small parametric deviations. They may include deviation of load sharing factors, load power factors, line parameters, and so on.

${ }^{2}$ We use the word "expectation" to imply the mathematical expectation instead of a subjective appreciation in this paper. 
- The steady state model of the power system is assumed. We are interested in the post contingency performance after an uncertain disturbance occurs.

- Some assumptions on the probability distribution are made. They include a Poisson distribution of contingencies, Multi-Variate-Normal distributions of parametric deviations and Normal distribution of load interruption voltages. The description of these distribution functions can be found in [1].

- The occurrence of contingencies are independent of each other, and they are also independent of other system parametric deviations and the operating condition. The individual parametric deviations, e.g., the real and reactive power deviations of bus loads, are considered correlated depending on the statistical data of these deviations. Other uncertainties outside the power system are assumed to be independently distributed.

- The impact of any contingency is assumed to include only the influence of voltage out-of-limit and its direct effect in terms of customer load interruption. We do not include any sympathetic effects which might lead to loss of additional components.

\section{B. Definition}

We define the "risk" as a condition under which there is a possibility of an adverse deviation from a desired outcome that is expected or hoped for [2].

There are two primitives included within this definition: $f u$ ture uncertainties and impact of outcomes.

Furthermore, we define the degree of risk ${ }^{3}$ as the expectation of the dollar based impacts of those outcomes. It would be the amount of impact multiplied by the corresponding probability of outcome. Expected value is a measure of risk in the theory of "risk management," although other measures, such as probability and variance, can be used to quantify the degree of risk [2].

\section{FRAMEWORK FOR RISK-BASED VOLTAGE ASSESSMENT}

We assume that there are two distinct outcomes for the future performance of system voltages, collapse or no collapse. The bifurcation point shown on the $P-V$ curve provides the boundary between these two outcomes. With the system operating without suffering a voltage collapse, the voltage may go below the tolerance of loads, resulting in load interruption. On the other hand, under some conditions, the system may approach a voltage collapse although all voltages are close to their nominal values.

Our general expression includes both of these risks,

$$
\begin{aligned}
\mathcal{R}\left(X_{0}\right)= & E\left[\mathcal{I} \mid X_{0}\right] \\
= & \mathcal{P}\left(\text { Collapse } \mid X_{0}\right) * E[\mathcal{I}(\text { Collapse })] \\
& +\left[1.0-\mathcal{P}\left(\text { Collapse } \mid X_{0}\right)\right] * E[\mathcal{I}(\text { No Collapse })]
\end{aligned}
$$

where $X_{0}$ stands for the current operating condition. This risk, $\mathcal{R}\left(X_{0}\right)$, depends on the probability of voltage collapse

\footnotetext{
${ }^{3}$ We will use the term "risk" to mean the degree of risk in the later sections.
}

$\mathcal{P}\left(\text { Collapse } \mid X_{0}\right)^{4}$ under the condition $X_{0}$, the expected impact of collapse $E[\mathcal{I}($ Collapse $)]$ and the expected impact of no collapse, $E[\mathcal{I}$ (No Collapse) $]$.

We will address each of these terms in the following sections. We drop the notation of the given operating condition $X_{0}$ in the following derivations for simplicity. The reader should be aware that all the derivations are based on $X_{0}$, i.e., all of the expressions are functions of $X_{0}$. This functional dependency, together with use of historical data, implies we are providing an expectation of the future using what we know about the present $\left(X_{0}\right)$ and the past (probability data).

\section{Probability of Voltage Collapse}

There are several uncertainties associated with the voltage collapse under the scope of short-term operating time frame. They are 1) contingencies, 2) short-term system load, 3) short-term parametric deviations, e.g., deviations of load sharing, generation dispatch, and other uncertainties if desired.

\section{A. Contingency}

The occurrence of a contingency, by assumption, follows a Poisson distribution, i.e.,

$$
E_{i} \sim \operatorname{Possion}\left(\lambda_{i} t\right)
$$

where the $\lambda_{i}$ is the occurrence rate of the contingency $E_{i}$, the time frame $t$ is the time used to estimate our future risk, i.e., we are assessing the operating risk within next $t$ hours.

\section{B. Short-Term Load Fluctuation}

Besides contingencies, the load drift and variation may be another uncertainty in the near future. A short-term load forecast provides an expectation of load $\mu_{L}$ and its standard deviation $\sigma_{L}$. By our assumption, it is normally distributed,

$$
L \sim N\left(\mu_{L}, \quad \sigma_{L}^{2}\right)
$$

\section{Short-Term Parametric Deviation}

In reality, the load sharing factors, load power factors, generation dispatch, and other system parameters will not be certain in the future, even though we may forecast or estimate them very well. So, we assume:

- The parameters are random in the future, and they follow a Multi-Variate-Normal (MVN) distribution around their expected values, and

- Their deviations, although random, are small such that linear approximation of maximum loadability with respect to these random parameters is valid.

Let us denote the expectation of these parameters as $E\left[\underline{K}_{P}\right]$, where the parametric column vector $\underline{K}_{P}$ may include all the possible system parameters, such as load sharing factors, generation dispatch, and so on.

\footnotetext{
${ }^{4}$ We use the notation $P(A \mid B)$ to represent the conditional probability of event $A$ under the given condition $B$. Similarly, $E[A]$ will be the expected value of $A$, and $E[A \mid B]$ is the conditional expectation of $A$ given $B$.
} 
Based on the given expectation of $E\left[\underline{K}_{\Gamma}\right]$, a Continuation Power Flow (CPF) [4] or other techniques will provide an expectation of maximum loadability $E\left[L_{m i}\right]$ and the margin sensitivities $\underline{S}_{P}$ with respect to these parameters ([5], [6]). Then,

$$
L_{m i}=E\left[L_{m i}\right]+\underline{S}_{P}^{T} \times\left(\underline{K}_{P}-E\left[\underline{K}_{P}\right]\right)
$$

where $L_{m i}$ is the system maximum loadability; it is random due to the random parameters $\underline{K}_{P}$. The MVN distribution of parameters $\underline{K}_{P}$ is,

$$
\underline{K}_{P} \sim \operatorname{MVN}\left(E\left[\underline{K}_{P}\right], \quad \mathbf{V}_{P}\right)
$$

where $E\left(\underline{K}_{P}\right)$ is the expectation of the system parametric scenario, and $\mathbf{V}_{P}$ is the variance-covariance matrix of these parameters. The elements of the variance-covariance matrix represent both the variance of each parameter and the correlation with respect to each other. This matrix can be estimated from the samples of historical data [3].

It can be proven that $L_{m i}$, a linear function of the MVN distributed $\underline{K}_{P}$, also follows a Normal distribution. Its expected value is $E\left[L_{m i}\right]$, and the variance is $\underline{S}_{P}^{T} \mathbf{V}_{P} \underline{S}_{P} .{ }^{5}$

The probability distribution of maximum loadability is therefore,

$$
L_{m i} \sim N\left(E\left[L_{m i}\right], \quad \underline{S}_{P}^{T} \mathbf{V}_{P} \underline{S}_{P}\right)
$$

and depends on the value of the parameters, their variability, and how they correlate with each other.

\section{Probability of Collapse}

Under a given topology determined by a contingency, when both the load level $L$ and the maximum loadability $L_{m i}$ are random, the probability of voltage collapse is the probability that the load margin $M_{i}=L_{m i}-L$ is negative. ${ }^{6}$ The probability distributions of $L$ and $L_{m i}$ are obtained through the expression (3) and (5), respectively. Since both are Normally distributed, the resultant load margin $M_{i}$ will be also Normal, with a mean of $\mu_{m i}$ and a variance of $\sigma_{m i}$. That is,

$$
\mathcal{P}\left(\text { collapse } \mid E_{i}\right)=\mathcal{P}\left(M_{i}<0 \mid E_{i}\right)
$$

where the random load margin $M_{i}$ has a Normal distribution.

$$
\begin{aligned}
M_{i} & \sim N\left(\mu_{m i}, \quad \sigma_{m i}^{2}\right) \\
\mu_{m i} & =E\left[L_{m i}\right]-\mu_{L} \\
\sigma_{m i}^{2} & =\underline{S}_{P}^{T} \mathbf{V}_{P} \underline{S}_{P}+\sigma_{L}^{2}
\end{aligned}
$$

By the Total Probability Theorem [1], the total probability of voltage collapse under the system exposed to uncertain contingencies is,

$$
\mathcal{P}(\text { collapse })=\sum_{E_{i}} \mathcal{P}\left(\text { collapse } \mid E_{i}\right) * \mathcal{P}\left(E_{i}\right)
$$

\footnotetext{
${ }^{5}$ Reference [3] has a thorough proof of the theory of linear models in statistics. ${ }^{6}$ We neglect the partial load curtailment when the load margin is still positive though this curtailment is required to prevent the "actual" voltage collapse in the current industry practice. We are more interested in obtaining the risk of voltage collapse if the curtailment would not be activated and making an informative curtailment decision based on the risk of collapse.
}

TABLE I

ELECTRIC SERVICE DEVIATION TOLERANCES FOR LOAD-AND-CONTROL EQUIPMENT

\begin{tabular}{l|l}
\hline \multicolumn{1}{c|}{ Device } & \multicolumn{1}{c}{ Voltage Level } \\
\hline Communication equipment & $\pm 5 \%$ \\
$\begin{array}{l}\text { Computers, } \\
\text { data processing equipment }\end{array}$ & $\pm 10 \%$ \\
Contactors, motor starts & \\
Lighting & $-15 \%$ to $+10 \%$ \\
$\quad$ Fluorescent & $-10 \%,-25 \%$ \\
Incandescent & $+18 \%$ \\
Motors, standard induction & $\pm 10 \%$ \\
Resistance loads, furnaces, heaters & Variable \\
$\ldots$ & \\
\hline
\end{tabular}

where the conditional probability $\mathcal{P}\left(\right.$ collapse $\left.\mid E_{i}\right)$ and the probability of contingency, $\mathcal{P}\left(E_{i}\right)$, are given by (6) and (2), respectively.

\section{IMPACT-WhEN VOLTAGE DOES Not COLlAPSE}

Under some conditions, voltages may decline to levels that result in partial load interruption, yet voltage collapse is avoided. This is the case we address in this section.

\section{A. Component-Based Load Interruption}

Under- or over-voltage protection is widely used in both power system distribution networks and the load itself. This protection is installed to protect the distribution components and the loads from over-current or other potential damage due to unacceptable load voltage. Additionally, load shedding schemes are used to prevent the system from cascading voltage collapse [7], [8]. These schemes will automatically trip the individual load or load groups under the condition that the voltage violates their set thresholds, leading to service interruption of tripped users. Also, some loads may drop off by themselves without any action of protective relays when voltage is unsustainable.

Table I [9], summarizes the examples of interruption voltages due to distribution protection.

Because the load interruption voltages are equipment dependent, they should be modeled individually. However, an entire distribution system is usually modeled as a single aggregated bus load in typical power flow and stability studies. This aggregated bus load is the composition of many individual loads with a number of different load characteristics.

Reference [10] presents a component-based method to specify an aggregated load model, where the load characteristic of interest is power sensitivity to voltage, rather than load interruption voltage. In this approach, the load mix is specified for each bus in terms of residential, commercial and industrial classes. ${ }^{7}$ This data can be derived from customer billing information.

This component-based approach is attractive because it provides for an upward aggregation of the available information,

\footnotetext{
${ }^{7}$ The load can be classified on a subclass level, like resistance heating, room air conditioner, lighting, water heating, and etc., if detailed information of load mix is obtained.
} 
i.e., the load class mix data, into the model to be used in the study. It also avoids the tedious work of modeling each individual load by grouping similar loads into class or subclass. The same approach can be used to model load interruption voltage.

We assume that the load mix configuration at a bus is specified [10]. Additionally, we further assume the distribution of interruption voltages within a load class $c$ is Normal. That is,

$$
V_{L, c} \sim N\left(\mu_{c}, \quad \sigma_{c}^{2}\right)
$$

where both the mean, $\mu_{c}$, and the standard deviation, $\sigma_{c}$, of an interruption voltage within a load class " $c$ " can be estimated through load statistics.

\section{B. Expected Impact on Load with a Given Voltage}

The service interruption at a "bus" occurs when the bus voltage is beyond the individual loads' sustainable range. With $K_{\text {bus, } c}$ as the percentage share of a load class $c$ at a particular bus, the impact on the interrupted load is its service interruption cost multiplied by its interruption amount.

$$
\begin{aligned}
& \mathcal{I}_{\text {bus }}\left(V_{\text {bus }}\right) \\
& \quad=P_{b u s} \sum_{c} C_{b u s, c} * K_{b u s, c} * I\left(V_{L, c}>V_{b u s} \mid V_{b u s}\right)
\end{aligned}
$$

where

$$
\begin{aligned}
& \text { Pbus } \\
& \text { Cbus,c } \\
& V_{L, c} \\
& I\left(V_{L, c}>V_{b u s} \mid V_{b u s}\right) \\
& E\left[\mathcal{I}_{\text {bus }} \mid V_{\text {bus }}\right] \\
& =E\left[P_{b u s}\right] \sum_{c} E\left[C_{b u s, c}\right] * K_{b u s, c} * \mathcal{P}\left(V_{L, c}>V_{b u s} \mid V_{b u s}\right)
\end{aligned}
$$

where the independence of $P_{b u s}, C_{b u s, c}$, and $V_{L, c}$ is used. $E\left[P_{b u s}\right]$ is the expected value of the forecasted load at the bus. The expectation of service interruption cost for each load class, $E\left[C_{b u s, c}\right]$, which includes the uncertainties of both interruption cost rate and interruption duration of a load class at a bus, is obtained by any regression method [3] based on customer survey or historical data. References [11] and [12] give a summary and survey on these cost evaluations. The probability term, $\mathcal{P}\left(V_{L, c}>V_{b u s} \mid V_{b u s}\right)$ is calculated from the Normal distribution of tolerance voltage, $V_{L, c}$, under the given load bus voltage $V_{b u s}$.

\section{Expected Impact with No-Voltage-Collapse}

Section V-B gives the expected impact with a given bus voltage. The bus voltage, however, depends on 1) contingen- cies, 2) short-term system load level, 3) short-term parametric deviations.

With small deviations of system parameters, a linear approximation of voltage around its expectation is assumed such that a Multi-Variate-Normal distribution of bus voltages is obtained.

$$
\begin{aligned}
& V=E\left[V \mid L, E_{i}\right]+\left(\frac{\partial \underline{V}}{\partial \underline{K}_{P}}\right) \times\left(\underline{K}_{P}-E\left[\underline{K}_{P}\right]\right) \\
& \underline{V} \sim \operatorname{MVN}\left(E\left[\underline{V} \mid L, E_{i}\right], \quad\left(\frac{\partial \underline{V}}{\partial \underline{K}_{P}}\right) \mathbf{V}_{P}\left(\frac{\partial \underline{V}}{\partial \underline{K}_{P}}\right)^{T}\right)
\end{aligned}
$$

where $\left(\partial \underline{V} / \partial \underline{K}_{P}\right)$ is the sensitivity matrix of bus voltages with respect to the variation of system parameters. If the voltage does not collapse, the expectation of bus voltages $E\left[\underline{V} \mid L, E_{i}\right]$ is obtained by solving the power flow based on the expected system condition and the contingency $E_{i} . \mathbf{V}_{P}$ is again the variance-covariance matrix of parametric deviations as defined in Section IV-C.

With the above Normal distribution of bus voltages, the expected voltage impact for the study system with a given load level and given contingency is,

$$
E\left[\mathcal{I} \mid L, E_{i}\right]=\sum_{b u s} \int_{V_{b u s}} E\left[\mathcal{I}_{b u s} \mid V_{b u s}\right] \mathcal{P}\left(V_{b u s}\right) d V_{b u s}
$$

where $E\left[\mathcal{I}_{\text {bus }} \mid V_{\text {bus }}\right]$ is defined in (11), and $\mathcal{P}\left(V_{\text {bus }}\right)$ is the Normal probability density function provided by (12).

Under the exposure to the uncertain load level and contingencies, the expected impact of voltage out-of-limits, when voltage does not collapse, is,

$$
\begin{aligned}
& E[\mathcal{I}(\text { No Collapse })] \\
& \quad=\sum_{E_{i}}\left(\int_{L} E\left[\mathcal{I} \mid L, E_{i}\right] * \mathcal{P}(L) d L\right) \mathcal{P}\left(E_{i}\right)
\end{aligned}
$$

which lumps all the possible contingencies ${ }^{8}$ and the load drifting. The probability of a load level, $\mathcal{P}(L)$, and a contingency, $\mathcal{P}\left(E_{i}\right)$, are given by the expression (3) and (2).

\section{IMPACT-When VOLTAGE COLlaPSES}

It is possible to mitigate the impact of voltage collapse via corrective or restorative operating actions. It is also possible that partial interruption can mitigate the voltage collapse and prevent full interruption. However, the effectiveness of these actions are very uncertain. Therefore we assume here that voltage collapse results in total system blackout.

The expected impact is then the interruption cost of the entire systems' load, i.e.,

$$
E[\mathcal{I}(\text { Collapse })]=\sum_{\text {bus }}\left(P_{\text {bus }} \sum_{c} C_{b u s, c} * K_{\text {bus }, c}\right)
$$

where all the loads in the system and all the load components at a bus are interrupted.

\footnotetext{
${ }^{8}$ Theoretically, one must include all contingencies here, but practically, one only includes the "credible" contingencies.
} 


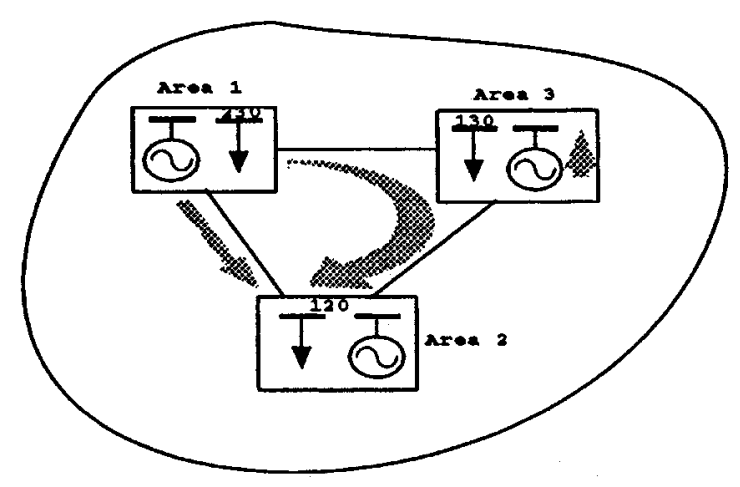

Fig. 2. Local illustration of IEEE-RTS 96.

TABLE II

LOADABILITY UNDER VARIOUS CONTINGENCY CONDITIONS

\begin{tabular}{l|lc}
\hline Contingency & $\begin{array}{l}\text { Occurrence } \\
\text { Probability }\end{array}$ & $\begin{array}{c}\text { Loadability } \\
(\times 100 \mathrm{MW})\end{array}$ \\
\hline No outages & 0.9999 & 40.70 \\
Out $130-120$ & $4.58 \times 10^{-5}$ & 39.14 \\
Out 230-130 & $4.58 \times 10^{-5}$ & 37.32 \\
Out 230-120 & $4.58 \times 10^{-5}$ & 36.89 \\
\hline
\end{tabular}

\section{EXAMPLES}

We provide an illustration of the proposed risk analysis on the IEEE Reliability Test System (IEEE-RTS 96) [13].

We have chosen a scenario where three contingencies, each one being an outage of a transmission line, provoke voltage collapse $^{9}$ on the load bus. An illustration of the system diagram is shown in (Fig. 2).

The time frame of interest is one hour. Under this time frame, we assume the forecasted expectation ${ }^{10}$ of the future system will be the same as the current operating condition. The standard deviation of this future load level is assumed to be $2 \%$. We further assume the deviation of load sharing factors on each bus to be the parametric variation which has $5 \%$ standard deviations around the expected values. The occurrence of contingencies are estimated from annual outage rates for the corresponding transmission lines. Both the probability of each contingency, including that of no-contingency condition which is $1.0-\sum_{E_{i}} \mathcal{P}\left(E_{i}\right)$, and the corresponding maximum loadability based on expected system parameters are listed in Table II.

The value of outage rate we assumed here is for simplicity and for showing the different effects even when the outage rates are same. One should improve the accuracy of these values by relating them to line length or actual historical data of each line.

\section{A. Probability of Collapse}

Suppose that the current load level is at $3600 \mathrm{MW}$, and the load sharing factors on each load bus are as listed in [13]. We wish to compute the probability of voltage collapse under the current operating condition.

${ }^{9}$ Here, we only consider the voltage problem. However, these contingencies may also cause thermal overload and transient instability in the system. Our generalized approach provides uniform measurement in assessing the composite risk associated with all three types of security problems. This attractive feature of the approach will be illustrated in another paper.

${ }^{10} \mathrm{We}$ emphasize that this is only an expectation because the future system will almost always deviate from this forecasted system.
TABLE III

RANDOMNESS OF LOADABILITY

\begin{tabular}{l|lcc}
\hline Contingency & $\begin{array}{l}\text { Occurrence } \\
\text { Probability }\end{array}$ & $\begin{array}{c}\text { Expected } \\
\text { Loadability } \\
(\times 100 \mathrm{MW})\end{array}$ & $\begin{array}{c}\text { Standard } \\
\text { Deviation }\end{array}$ \\
\hline No outages & 0.9999 & 40.70 & 0.3839 \\
Out 130-120 & $4.58 \times 10^{-5}$ & 39.14 & 0.4179 \\
Out 230-130 & $4.58 \times 10^{-5}$ & 37.32 & 0.3970 \\
Out 230-120 & $4.58 \times 10^{-5}$ & 36.89 & 0.3353 \\
\hline
\end{tabular}

TABLE IV

RANDOMNESS OF LOAD MARGIN

\begin{tabular}{l|cc|l}
\hline Contingency & $\begin{array}{c}\text { Expected } \\
\text { Margin } \\
(\times 100 \mathrm{MW})\end{array}$ & $\begin{array}{l}\text { Standard } \\
\text { Deviation }\end{array}$ & $\begin{array}{l}\text { Probability } \\
\text { of Collapse } \\
\text { w/ Contingency }\end{array}$ \\
\hline No outages & 4.70 & 0.8160 & $4.3 \times 10^{-9}$ \\
Out 130-120 & 3.14 & 0.8325 & $8.0 \times 10^{-5}$ \\
Out 230-130 & 1.32 & 0.8225 & 0.0547 \\
Out 230-120 & 0.89 & 0.7942 & 0.1306 \\
\hline
\end{tabular}

With $2 \%$ standard deviation, the true load has $95 \%$ probability of fluctuating within an interval of $3600 \pm 1.96 \times 72 \mathrm{MW}$.

\begin{tabular}{|c|c|}
\hline Expected Load & Standard Deviation \\
\hline $36.00 \times 100 \mathrm{MW}$ & $0.72 \times 100 \mathrm{MW}$ \\
\hline
\end{tabular}

Moreover, the $5 \%$ standard deviations of load sharing factors cause the $P-V$ curve or the maximum loadability to be uncertain. Both the randomness of the loadability and the corresponding contingency are listed in Table III. They are obtained from the expression (5).

The load margins between the random maximum loadability and the random load level for each contingency, by the (7), are given in Table IV.

The probability of collapse over the next hour, for the load being $3600 \mathrm{MW}$, is therefore only $8.5 \times 10^{-6}$. It is calculated by summing up all the products of collapse probability under contingency and the probability of the corresponding contingency, i.e., $0.9999 * 4.3 \times 10^{-9}+4.58 \times 10^{-5} * 8.0 \times 10^{-5}+4.58 \times 10^{-5}$ $* 0.0547+4.58 \times 10^{-5} * 0.1306$. Fig. 3 provides a plot of collapse probabilities against different loading levels for each contingency (including "no outage"). We also show the total probability which is the sum of the collapse probabilities weighted by the contingency probabilities.

\section{B. Expected Impact}

For a simple illustration, we assume all the load buses have identical load class mix say $100 \%$ residential load with mean interruption voltage at 0.85 (lower mean), 1.15 (upper mean), and a 0.02 of standard deviation.

The expected portion of load interrupted at a bus with various voltage levels is computed in Fig. 4, where the base for the per unit values is the expected amount of load at that bus.

It is possible that voltage may be stable, yet the voltage may decline below the load's tolerable range. The expected amount of load interrupted at bus 120 under different load levels is shown in Fig. 5. This result is obtained by combining ${ }^{11}$ both

\footnotetext{
${ }^{11}$ Given a load level, we will have a voltage level from Fig. 1. This voltage, from the Fig. 4, will lead to some amount of load interruption. Therefore, we have the plot in Fig. 5.
} 


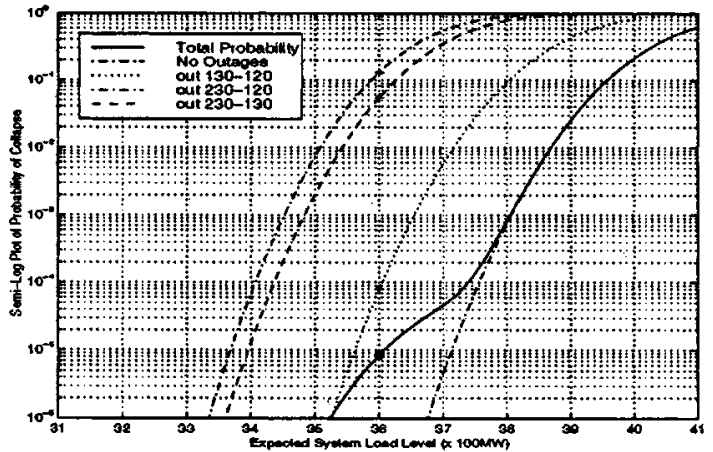

Fig. 3. Probability of collapse.

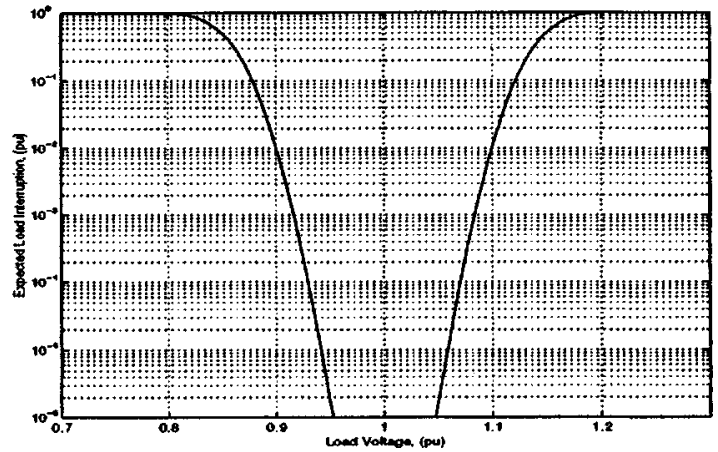

Fig. 4. Expected load interrupted by various voltages.

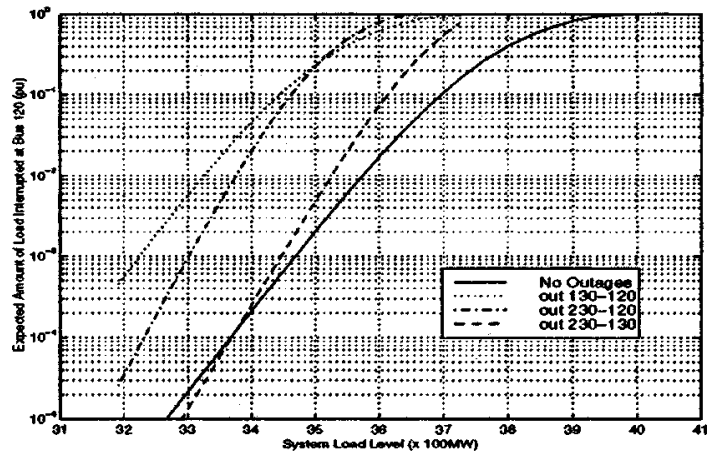

Fig. 5. Expected load interruption.

the expected $P-V$ curve in Fig. 1 and the expected interruptionvoltage curve in Fig. 4.

The expected impact of service interruption when voltage does not collapse is depicted in Fig. 6, ${ }^{12}$ where an expected cost of $\$ 50$ per MWhour $\times 6$ hours service interruption is uniformly assumed for the entire system. The impact curve in the figure suggests the potential cost of load interruption due to voltage out-of-limit under the condition that the system does not suffer the voltage collapse problem. It represents the term $E[\mathcal{I}($ No Collapse $)]$ in (1).

For the impact of collapse, we assume the outcome will be an entire system blackout. The cost is also uniformly assumed as $\$ 50$ per MWhour for an expected interruption of 6 hours. When

\footnotetext{
${ }^{12}$ Because of the low probability of contingency in this example, the expected impact is dominated by the no-contingency case, and the two curves are indistinguishable.
}

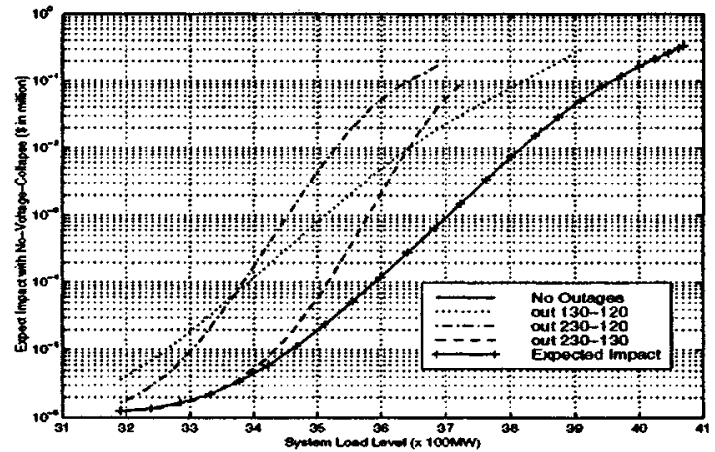

Fig. 6. Impact-When voltage does not collapse.

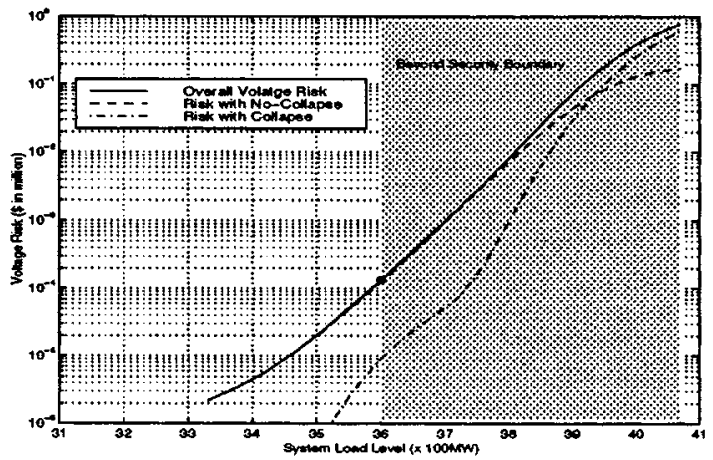

Fig. 7. Semilog plot of risk.

load level is at $3600 \mathrm{MW}$, this impact is expected to be $\$ 1.08$ million.

\section{The Risk of Voltage Assessment}

By equation (1), a semilog plot of risk associated with voltage problems is depicted in Fig. 7. It is the sum of two parts: risk of collapse and risk of voltage out-of-limit with no-collapse.

The left boundary of the shaded area in the figure is the worst-case single contingency security boundary. This is the traditional firm security limit for this system. The right boundary of the shaded area is the no-contingency limit. The solid curve of total risk indicates that the expected impact of voltage security problems, voltage collapse and out-of-limit, varies with the different operating conditions, the load levels in this case.

\section{DISCUSSION}

The risk in Fig. 7 gives a quantitative measurement of reliability for the operating positions both within the traditional boundary and outside the boundary. It is useful as a decision making aid in determining operating limits associated with voltage problems. For example, one might compare the risk of the deterministic limit given in Fig. 7, which is approximately $\$ 135$ over the next hour, with the risk associated with other 


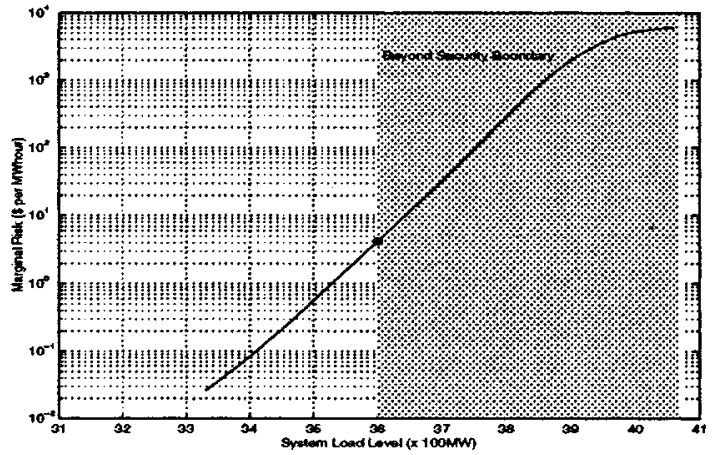

Fig. 8. Semilog plot of marginal risk.

deterministic limits, to assess the uniformity of deterministic limits in terms of risk.

The risk provides a "leading indicator" of reliability trend under the current operating condition. It is not only an "alarm signal" which monitors the security level of the current system, but it also provides a uniform security measurement for the future, based on the current information. Fig. 7 provides the risk over the next hour associated with a certain forecasted expectation, with standard deviation in load and other parameters characterizing the operating condition. The approach provides that we can also extend the time frame using an appropriate forecasting technique to obtain the risk variation with time.

Reliability Has a Price: Instead of limiting the operating condition with significant reliability margin inside a deterministic boundary, the "risk" suggests a conceptual price of reliability. The risk implies an expectation of future cost due to possible reliability problems. It adds an additional implicit cost to the cost of energy delivered. Fig. 8 plots a marginal risk with respect to various loading positions. For example, it suggests an expected $\$ 4.25$ per MWhour of additional implicit cost charged for the possibility of losing voltage security when the system is running at the level of $3600 \mathrm{MW}$. Also the idea of "Bus Incremental Risk"13 can be introduced to price the cost of security at each bus.

This paper focuses on the risk of voltage insecurity. It is possible to extend the concept to other security problems, such as thermal overload and transient instability. The uniformity of the risk provides that we can construct a "composite risk index" for all of the concerned security problems. The detailed description of composite risk will be given in another paper. The reader can refer to [14]-[17] for the risk analysis of thermal overload and transient instability.

The probabilistic method to assessing the risk of voltage collapse in this paper is based on sensitivities of load margin around the bifurcation point [5], [6]. Here, we assume the risk calculation is being done for a near term future, and consequently, changes in operating conditions are small. Voltage risk assessment for futures significantly different from the present should avoid use of linearized sensitivities.

The risk, the expectation of impact, discussed in this paper, however, only provides an expectation of future insecurity cost. It does not guarantee the future outcome will be exactly the same as this statistical expectation. More information, such as vari-

${ }^{13}$ This comes from the pricing method of "Bus Incremental Cost (BIC)." ance of this risk, should be included together with risk to make better operation decisions.

\section{CONCLUSION}

A probabilistic method to compute the operating risk of voltage collapse and voltage out of limits is presented in this paper. The resulting risk represents the expected future cost of voltage insecurity based on information from the current operating condition. The risk gives a quantitative measure of reliability both within and outside the traditional security boundary. It is promising in

- quantifying a composite risk in hybrid security problems,

- providing a "leading indicator" for reliability, and

- pricing power system security.

\section{REFERENCES}

[1] G. Casella and R. L. Berger, Statistical Inference. Pacific Grove, CA: Brooks/Cole Pub. Co., 1990.

[2] E. J. Vaughan, Risk Management. New York, NY: John Wiley \& Sons, c1997, pp. 8-11.

[3] S. R. Searle, Linear Models. New York, NY: John Wiley \& Sons, c1971, pp. 40-44.

[4] V. Ajjarapu and C. Christy, "The continuation power flow: A tool for steady state voltage stability analysis," IEEE Trans. on Power Systems, vol. 7, no. 1, pp. 416-423, Feb. 1992.

[5] B. Long and V. Ajjarapu, "The sparse formulation of ISPS and its application to voltage stability margin sensitivity and estimation," IEEE Trans. on Power Systems, to be published.

[6] S. Greene, I. Dobson, and F. L. Alvarado, "Sensitivity of the loading margin to voltage collapse with respect to arbitrary parameters," IEEE Trans. on Power Systems, vol. 12, no. 1, pp. 262-272, Feb. 1997.

[7] "Summary of 'system protection and voltage stability'," IEEE Trans. on Power Delivery, vol. 10, no. 2, pp. 631-637, Apr. 1995.

[8] C. W. Taylor, Power System Voltage Stability. New York, NY: McGraw-Hill, c1994, pp. 67-92.

[9] Electrical Distribution-System Protection, 3rd ed: Cooper Power Systems, 1990, p. 250.

[10] W. W. Price, K. A. Wirgau, A. Murdoch, J. V. Mitsche, E. Vaahedi, and M. A. Elkady, "Load modeling for power flow and transient stability computer studies," IEEE Trans. on Power Systems, vol. 3, no. 1, pp. 180-187, Feb. 1988.

[11] "The national electric reliability study, final report," U.S. Dept. of Energy, Washington, DC, DOE/EP-0004, Dist. Category UC-97C, Apr. 1981.

[12] "Cost-benefit analysis of power system reliability: Determination of interruption costs,", EPRI EL-6791, vol. 1, Project 2878-1, Final Report, Apr. 1990.

[13] Reliability Test System Task Force of the Application of Probability Methods Subcommittee, "The IEEE reliability test system—1996,", 96 WM 326-9 PWRS.

[14] H. Wan, J. D. McCalley, and V. Vittal, "Increasing thermal rating by risk analysis," IEEE Trans. on Power Systems, to be published.

[15] J. D. McCalley, A. Fouad, V. Vittal, A. Irizarry-Rivera, B. Agrawal, and R. Farmer, "A risk based security index for determining operating limits in stability-limited electric power systems," IEEE Trans. on Power Systems, vol. 12, pp. 1210-1219, Aug. 1997.

[16] V. Vittal, J. McCalley, V. Van Acker, W. Fu, and N. Abi-Samra, "Transient instability risk assessment," in Proc. Of the 1999 IEEE PES Summer Meeting, July 18-22, 1999, to be published.

[17] V. Van Acker, J. McCalley, and V. Vittal, "Risk based transient instability assessment," in 1999 Proc. of Power Tech., Budapest, Hungary, Aug. 1999 , to be published. 
Hua Wan received his B.S. (1992) and M.S. (1995) degrees in electrical engineering from the Southeast University, Nanjing, China. After being employed by the Nanjing Automation Research Institute, China, he is currently working toward a Ph.D. in electrical engineering at Iowa State University. His interests include risk management, statistics, probabilistic methods, and power system security assessment. He is a Student Member of the IEEE.

James D. McCalley is an Associate Professor of Electrical and Computer Engineering at Iowa State University, where he has been employed since 1992. He worked for Pacific Gas and Electric Company from 1986 to 1990. Dr. McCalley received the B.S. (1982), M.S. (1986), and Ph.D. (1992) degrees in electrical engineering from Georgia Tech. He is a registered Professional Engineer in California and a Senior Member of the IEEE.
Vijay Vittal is Professor of the Electrical and Computer Engineering at Iowa State University. He received the B.E. (1977) in electrical engineering from Bangalore, India, the M.Tech. (1979) from the Indian Institute of Technology, Kanpur, India, and the Ph.D. (1982) from Iowa State University, Ames, IA. He is the recipient of the 1985 Presidential Young Investigator Award. He is a Fellow of the IEEE. 